\title{
Phase Separation Phenomena in Solutions of Poly(2,6-Dimethyl-1,4-Phenylene Oxide). IV. Thermodynamic Parameters for Solutions in a Series of Homologous Solvents: Toluene to Hexylbenzene
}

\author{
D. M. KOENHEN,** A. BAKKER, L. BROENS, J. W. A. VAN DEN \\ BERG,$^{\dagger}$ and C. A. SMOLDERS, Twente University of Technology, \\ Department of Chemical Technology, P.O. Box 217, 7500 AE Enschede, \\ The Netherlands
}

\begin{abstract}
Synopsis
Melting-point curves for solutions of poly(2,6-dimethyl-1,4-phenylene oxide) (PPO) in a series of homologous solvents (toluene to $n$-hexylbenzene) have been obtained from visual and differential scanning calorimetry measurements. The measured melting points were used to calculate thermodynamic interaction parameters. It was found that consistent values were obtained with the Flory-Hoffman melting-point depression equation, if the assumption was made that solvent molecules are incorporated in the crystal lattice. To this end, an adapted dependence of the enthalpy of melting per polymer unit on the cocrystallizing solvent was used. The values of the thermodynamic interaction parameters in the series of solvents and their dependence on polymer weight fraction are explained qualitatively with simplified versions of equation-of-state theory and solubility parameter theory.
\end{abstract}

\section{INTRODUCTION}

It has been shown in previous papers in this series that the phase separation of solutions of $\mathrm{PPO} \neq$ in toluene is a crystallization phenomenon. ${ }^{1-3}$ Measurements of the interaction parameter $g$ for these solutions showed a linear increase in $g$ with polymer concentration. ${ }^{1}$ The role of the solvent in the crystallization has not been made clear in previous work.

Thermodynamic parameters such as $g$ and $\Delta h_{u}^{0}$ (the enthalpy of melting per monomer) can be derived from melting-point curves. This presents an opportunity to test the hypotheses that may be put forward for the role of the solvent. It allows the determination of thermodynamic parameters for other solvent systems as well.

In this contribution, the melting-point curves have been determined for PPO in the solvents toluene, ethylbenzene, $n$-propylbenzene, $n$-butylbenzene, $n$-pentylbenzene, and $n$-hexylbenzene. These solvents are characterized by an increasing length of hydrocarbon chain on an aromatic phenyl ring. Normal alkanes are nonsolvents for PPO, whereas aromatics are

* Present address: Promac Nederland B.V., Zaltbommel, The Netherlands.

† To whom all correspondence should be addressed.

† Registered trademark of General Electric Company.

Journal of Polymer Science: Polymer Physics Edition, Vol. 22, 2145-2157 (1984) (c) 1984 John Wiley \& Sons, Inc.

CCC 0098-1273/84/122145-13\$04.00 
known as good solvents. ${ }^{4}$ Thus, in the series mentioned the solvent power decreases. This will of course affect the measured melting-point curves and the thermodynamic parameters.

There are also consequences which can be drawn from the model of the crystals assumed. If the solvent does not enter the crystal lattice, the usual melting-point theories can be applied. If the solvent forms part of the crystalline lattice, as is the case with solvents such as $\alpha$-pinene and decalin, ${ }^{5}$ the theory has to be adapted.

The purpose of this work was to see whether the thermodynamic parameters ( $\Delta h_{u}^{0}, g$, and $T_{m}^{0}$, the polymer melting point) determined for PPOtoluene solutions, in combination with the measured melting point curves for the homologous solvent series, could give a meaningful set of $g$ parameters for the series of solvents. Whether this set is indeed meaningful has been tested by comparing the experimental values of the $g$ parameters, derived from the melting-point curves, to values calculated from an equation-of-state theory and a solubility parameter theory.

\section{THEORY}

\section{The Thermodynamic Interaction Parameter $g$}

In order to describe polymer solution thermodynamics, use is made of the well-known Flory-Huggins equation, ${ }^{6}$

$$
\frac{\Delta G_{m}}{R T}=w_{0} \ln w_{0}+\sum \frac{M_{0}}{M_{i}} w_{i} \ln w_{i}+g w_{0} \sum w_{i}
$$

where $\Delta G_{m}$ is the free enthalphy of mixing for $M_{0}$ grams of solution, $T$ is the temperature in degrees Kelvin, $R$ is the universal gas constant, $w_{0}$ and $w_{i}$ are the weight fractions of solvent and polymer species, respectively, $M_{0}$ and $M_{i}$ are the molecular weight of solvent and polymer, and $g$ is the free enthalpy correction parameter.

This equation is given here in terms of weight fractions as was proposed by Scholte. ${ }^{7}$ The use of weight fractions is especially recommended when studying the same solution at different temperatures, because this concentration variable does not change with temperature as is the case with volume fractions. However, the original Flory-Huggins theory was derived on the basis of the lattice theory. ${ }^{6}$ This means that every segment in the lattice is associated with a certain volume, hence volume fractions should be used.

For organic substances the molecular weight of a fixed volume segment generally is approximately the same. ${ }^{8}$ This allows eq. (1) to be used. In view of the approximation that the same lattice is used for both the polymer and the solvent this equation probably gives a configurational entropy which is somewhat too high.

A second contribution comes from free-volume effects. For this type of correction the corresponding-states theories of Prigogine ${ }^{9}$ and Flory ${ }^{10}$ apply. Another reason for deviations from the Flory-Huggins configurational entropy is polymer chain flexibility and back bending. It is customary to correct for all nonidealities with the correction parameter $g$ in eq. (1) introduced in this form by Koningsveld. ${ }^{11}$ 


\section{Melting-point Depression}

The difference in chemical potential between a crystalline polymer segment and a polymer segment in the pure liquid state can be written as ${ }^{12}$

$$
\mu_{u}^{c}-\mu_{u}^{0}=-\Delta g_{u}^{0}=-\left(\Delta h_{u}^{0}-T \Delta s_{u}^{0}\right)
$$

where the unit is defined as the polymer segment and $\Delta g_{u}^{0}, \Delta h_{u}^{0}$, and $\Delta s_{u}^{0}$ are the partial molar free enthalpy, enthalpy of fusion and entropy of fusion per mole of pure polymer segment, respectively.

When we now put $\Delta h_{u}^{0} / \Delta s_{u}^{0}$ as $T_{m}^{0}$, this equation can be written as

$$
\mu_{u}^{c}-\mu_{u}^{0}=-\Delta h_{u}^{0}\left(1-T / T_{m}^{0}\right)
$$

The difference in chemical potential between a polymer unit in the solution and a polymer segment in the pure liquid state is given by ( $w=$ $\left.\Sigma w_{i}\right)$

$$
\begin{aligned}
\mu-\mu^{0}=R T\left[\frac{M_{0}}{M_{n}} \ln w+(1-w)\right. & \frac{M_{0}}{M_{n}}- \\
& \left.(1-w)+\left(g_{0}+\frac{g_{1}}{T}+2 g_{2} w\right)(1-w)^{2}\right]
\end{aligned}
$$

In this form this quantity is defined for $M_{0}$ grams as being one mole. Hence, for the difference in chemical potential per mole of polymer segment units, it should read

$$
\begin{aligned}
\mu_{u}-\mu_{u}^{0}=R T\left[\frac{M_{u}}{\bar{M}_{n}} \ln w+\right. & \frac{M_{u}}{\bar{M}_{n}}(1-w)- \\
& \left.(1-w) \frac{M_{u}}{M_{0}}+\frac{M_{u}}{M_{0}}\left(g_{0}+\frac{g_{1}}{T}+2 g_{2} w\right)(1-w)^{2}\right]
\end{aligned}
$$

In the latter two equations the correction parameter $g$ has been written as $g=g_{0}+g_{1} / T+g_{2} w$, where $g_{0}, g_{1}$, and $g_{2}$ are constants. ${ }^{11}$

In the equilibrium state between polymer crystals and the polymer solution the following relation is valid:

$$
\mu_{u}^{c}-\mu_{u}^{0}=\mu_{u}-\mu_{u}^{0}
$$

Therefore we obtain as an expression for the melting-point epression

$$
\begin{aligned}
\frac{1}{T_{m}}-\frac{1}{T_{m}^{0}}=\frac{-R}{\Delta h_{u}^{0}}\left[\frac{M_{u}}{\bar{M}_{n}} \ln w+\right. & (1-w) \frac{M_{u}}{\bar{M}_{n}}- \\
& \left.(1-w) \frac{M_{u}}{M_{0}}+\frac{M_{u}}{M_{0}}\left(g_{0}+\frac{g_{1}}{T}+2 g_{2} w\right)(1-w)^{2}\right]
\end{aligned}
$$


In the derivation of this equation the assumption has been made that $\Delta h_{u}$ and $\Delta s_{u}$ in eq. (2) do not depend on temperature, which is not correct. This problem has been solved by Hoffman, ${ }^{13}$ who showed that a correction term $T_{m} / T_{m}^{0}$ was necessary for the description of the chemical potential difference $\mu_{u}^{c}-\mu_{u}^{0}$ to correct for the temperature dependence of $\Delta h_{u}$ and $\Delta s_{u}$ below $T_{m}^{0}$. This results in the following equation for the lowering of the melting point:

$$
\begin{aligned}
\frac{\Delta h_{u}^{0}\left(T_{m}^{0}-T_{m}\right) T_{m}}{\left(T_{m}^{0}\right)^{2}}=- & R T_{m}\left[\frac{M_{u}}{\bar{M}_{n}} \ln w+(1-w) \frac{M_{u}}{\bar{M}_{n}}\right. \\
& \left.-(1-w) \frac{M_{u}}{M_{0}}+\frac{M_{u}}{M_{0}}\left(g_{0}+\frac{g_{1}}{T}+2 g_{2} w\right)(1-w)^{2}\right]
\end{aligned}
$$

So far the influence of crystal morphology has not been mentioned. Factors involving the crystal morphology have been evaluated for a similar system by Helms. ${ }^{14} \mathrm{He}$ found that the crystal morphology (surface enthalpy coordination numbers, fold length, etc.) has little influence on the thermodynamic parameter $g$. On the other hand, the characteristic melting enthalpy and thermodynamic melting point are affected.

Since we do not have specific knowledge about the crystal morphology as such in the systems considered here, we use eqs. (7) and (8) and we regard $\Delta h_{u}^{0}$ and $T_{m}^{0}$ as characteristic values for a given morphology and not as thermodynamic equilibrium values. This is justified when the factors involved in the morphology do not change too much in the series studied.

\section{Solvent Cocrystallization}

When solvent enters the crystal lattice the situation becomes somewhat more complicated. It has been assumed that the solvent enters the crystal lattice at a fixed ratio, between solvent molecules and polymer segment units (solvent may stabilize helix formation). It is known that the crystallinity in these systems disappears on removal of the solvent by evaporation. ${ }^{5}$

At the melting point, the crystals (including the solvent) will be in equilibrium with the solution in which the crystal is immersed. When nearly all crystals have been melted, the concentration is effectively the net solution concentration. Theoretically it is possible to obtain a certain (though very low) degree of crystallinity until the solution concentration approaches $100 \%$.

The chemical potential of the solution is again given by eq. (5) and the chemical potential of the crystal is again given by eq. (3), and with the Hoffman correction, eq. (8) is obtained again. The parameters $\Delta h_{u}^{0}$ and $T_{m}^{0}$ of course are now characteristic for a given polymer (solvent) crystal and they will change with a change of solvent.

It is to be expected that $\Delta h_{u}^{0}$ decreases with increasing molecular weight (i.e., volume) of the solvent because the crystals obtained will have larger spacings per unit cell. Even if the total melt enthalpy per gram of crystals were to remain constant, $\Delta h_{u}^{0}$ would decrease because this value is defined per mole of polymer units. 
Of course the total enthalpy of melting depends also on intermolecular forces and on helix stability, but it is assumed here that the variations over the solvent series of these last effects do not exceed the first-mentioned volume effect.

The change of $\Delta h_{u}^{0}$ with solvent can be modeled for a homologous series of solvents by the following reasoning. First, we assume that there is a solvent-dependent melting point $\left(T_{m}^{0}\right)^{\prime}$, which is a constant for the whole series. Second, we assume that a fixed amount of solvent molecules incorporate into the lattice, such that the polymer weight fraction $w$ of the pure polymer does not equal but is slightly less than unity. Our third assumption is that this amount, as well as the interaction energy between incorporated solvent molecules and crystals lattice, is independent of solvent molecular weight. Then, inserting $\left(T_{m}^{0}\right)^{\prime}$ for $T_{m}$ in eq. (8), we find as a first approximation

$$
\Delta h_{u}^{0}=\text { (const) } M_{u} / M_{0}
$$

[because of the supposed lack of dependence of $w$ and of $g$ parameters on solvent molecular weight; also note that the logarithmic term on the righthand side of eq. (8) cancels the second term for $w \rightarrow 1.0]$.

Usually (see ref. 1 and the Discussion section below) the enthalpy of fusion is expressed per gram of polymer units:

$$
\Delta h_{g}^{0}=\Delta h_{u}^{0} / M_{u}
$$

and so, by virtue of eq. (9), we have for the homologous series of solvents in question

$$
\Delta h_{g}^{0}=\text { const } / M_{0}
$$

Once we have determined $\Delta h_{g}^{0}$ from the experimental melting-point depression curve of the PPO-toluene system (with $M_{0}=92$ ), we know the constant in this equation. Then we have, for the homologous series of solvents toluene through $n$-hexylbenzene:

$$
\left(\Delta h_{g}^{0}\right)_{\text {solvent }}=\left(\Delta h_{g}^{0}\right)_{\text {toluene }} \times 92 / M_{0}
$$

This equation will be applied in the next section, and its consequences for the determination of $g_{0}$ and $g_{2}$ by means of eq. (8) are studied there for each solvent other than toluene.

In the Discussion section we compare our results for $\left(\Delta h_{g}^{0}\right)_{\text {toluene }}(11.84 \mathrm{cal} /$ g) with previous results. To this end, we note that some of those have been obtained from an equation for the melting-point depression based on eq. (4), and not on eq. (5). Inspection of these equations shows that our present $\Delta h_{g}^{0}$, based on eq. (8) and therefore on eq. (5), can be found from the enthalpies calculated previously by multiplying the latter by $M_{u} / M_{0}$. It should be stressed, however, that this correction is based on pure thermodynamics, and has nothing to do with the rough solvent incorporation model underlying eqs. (9) and (10). 


\section{EXPERIMENTAL}

\section{Visual Determinations of Melting Points}

Appropriate amounts of polymer and solvents were weighed into a Pyrex tube with an inner diameter of $3 \mathrm{~mm}$. The contents were degassed and sealed as previously described. ${ }^{1}$ The sealed tubes were heated to a temperature at which the contents became homogeneous. The homogeneous solutions were cooled by only $1^{\circ} \mathrm{C}$ per day to obtain crystals of the highest possible order. Then the tubes were heated again at a rate of $1^{\circ} \mathrm{C} /$ day and the melting points were detected as the point of disappearance of turbidity.

\section{DSC Determinations of the Melting Points}

The determinations were performed with a Perkin-Elmer DSC-1B differential scanning calorimeter. The solutions prepared as described above were quickly cooled to obtain a solid mass. The tubes were broken and a small amount ( $\pm 10 \mathrm{mg}$ ) of the solid mass was transferred to the liquidtype sample pans and sealed. The sample pans were heated to a temperature at which complete dissolution was assured, held 1 day at that temperature, and checked for weight loss. The sample pans, which had a good seal, were cooled down to room temperature at a rate of $1^{\circ} \mathrm{C}$ per $2 \mathrm{~h}$. The melting points were then detected by the end of the melting endotherm in the heating thermogram. The scanning speed was $16^{\circ} \mathrm{C} / \mathrm{min}$.

\section{Materials}

Determinations were made with polymer sample 1 , characterized by $\bar{M}_{n}=23,000, \bar{M}_{w}=44,000$.

The solvents were of analytical grade.

\section{RESULTS}

The results for the melting-point curves are given in Table I and in Figure 1. Visual and DSC determinations agreed to within $1^{\circ} \mathrm{C}$. As was expected the melting curves for the solvents with increasing length of hydrocarbon chain shift to higher temperatures.

For PPO solutions in toluene $g_{0}, g_{1}$, and $g_{2}$ values were evaluated by an independent method (light scattering) in a previous paper. ${ }^{1}$ This resulted in the values $g_{0}=0.58, g_{1}=0$, and $g_{2}=0.19$.

Literature values for $\Delta h_{u}^{0}$ and $T_{m}^{0}$ for PPO crystals show a broad range of values. ${ }^{1}$ Therefore we decided to evaluate these values directly from the melting-point curve in toluene. With a computer optimization program using eq. (8), the values $g_{0}=0.58, g_{1}=0, g_{2}=0.19$, and the measured toluene melting-point curve, we obtained $\Delta h_{g}^{0}=\Delta h_{u}^{0} / M_{u}=11.84 \mathrm{cal} / \mathrm{g}$. The optimum in the calculations is that value of $T_{m}^{0}$ at which $\Delta h_{u}^{0}$ is a constant over the $T_{m}$ vs. $w$ curve. Thus $T_{m}^{0}$ was found to be $580 \mathrm{~K}$. The same program was run for eq. (7) (the simple Flory equation), but we found unrealistically high $T_{m}^{0}$ values, coupled with very low $\Delta h_{u}^{0}$ values.

The above values found for $\Delta h_{u}^{0}$ and $T_{m}^{0}$ were then used to calculate the term $g_{0}+2 g_{2} w$ from eq. (8) for the other solvents, by using the experimental 
TABLE I

Melting-Point Depression $\left({ }^{\circ} \mathrm{C}\right)$ for PPO in the Homologous Alkylbenzene Solvent Series

\begin{tabular}{ccccccc}
\hline \multicolumn{1}{c}{$w^{\mathrm{a}}$} & Toluene & $\begin{array}{c}\text { Ethyl- } \\
\text { benzene }\end{array}$ & $\begin{array}{c}n \text {-Propyl- } \\
\text { benzene }\end{array}$ & $\begin{array}{c}n \text {-Butyl- } \\
\text { benzene }\end{array}$ & $\begin{array}{c}n \text {-Pentyl- } \\
\text { benzene }\end{array}$ & $\begin{array}{c}n \text {-Hexyl- } \\
\text { benzene }\end{array}$ \\
\hline 0.050 & 50.2 & 65.6 & 85.0 & 111.1 & 122.9 & 133.7 \\
0.105 & 56.9 & 73.3 & 92.7 & 116.8 & 128.0 & 137.2 \\
0.200 & 72.2 & 88.6 & 105.0 & 126.5 & 138.3 & 145.9 \\
0.305 & 87.1 & 101.9 & 118.3 & 136.2 & 147.0 & 154.1 \\
0.400 & 100.9 & 114.2 & 130.6 & 145.9 & 154.6 & 161.8 \\
0.495 & 116.8 & 126.5 & 143.4 & 155.2 & 161.8 & 168.5 \\
\hline
\end{tabular}

a Weight fraction of polymer.

melting points. From melting-point curves it is difficult to discriminate between the terms $g_{1} / T$ and $g_{2} w$; light-scattering measurements in the case of toluene solutions showed that $g_{1} / T \rightarrow 0$. For the sake of simplicity $g_{1} /$ $T$ was set to zero for the complete series of solvents and for the whole range of temperatures. We shall revert to this simplification in the Discussion section.

The results for $g_{0}+2 g_{2} w$ vs. $w$ are presented in Figure 2. As can be seen here, there is no linear relation between $g_{0}+2 g_{2} w$ and concentration $w$ as was found for toluene. This means that for solvents other than toluene consistent sets of $g_{0}$ and $g_{2}$ values cannot be calculated. Moreover, the values for $g_{0}+2 g_{2} w$ are surprisingly low.

In our analysis, involving participation of solvent in the crystal lattice

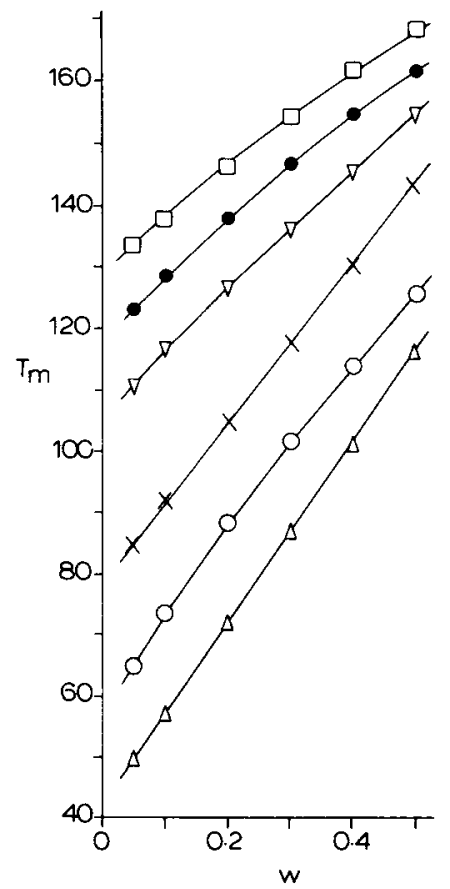

Fig. 1. Melting point curves for PPO in the homologous alkylbenzene solvent series: $(\triangle)$ toluene, $(\bigcirc)$ ethylbenzene, $(\mathrm{X}) n$-propylbenzene, $(\nabla) n$-butylbenzene, $(\odot) n$-pentylbenzene, and () $n$-hexylbenzene. 


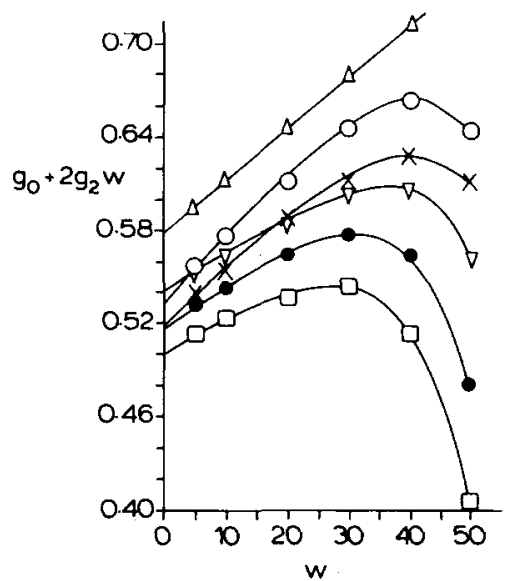

Fig. 2. Calculated curves for $\left(g_{0}+2 g_{2} w\right)$ for pure polymer crystals: points identified as in Figure 1.

of the polymer, an assumption has been made concerning the dependence of $\Delta h_{u}^{0}$ and $T_{m}^{0}$ on the molecular weight $M_{0}$ of the solvent. Since the $g$ values themselves are sensitive to $\Delta h_{u}^{0}$ and less sensitive to $T_{m}^{0}$, it is assumed that only $\Delta h_{u}^{0}$ (and therefore $\Delta h_{g}^{0}$ ) will decrease proportionally with increasing molecular weight of the solvent according to eqs. (9) and 10), whereas $T_{m}^{0}$ will remain constant at $580 \mathrm{~K}$. For toluene it was found that $\Delta h_{g}^{0}=11.84$ (cal/g); therefore for another solvent we have

$$
\triangle h_{g}^{0}=11.84 \times 92 / M_{0} \mathrm{cal} / \mathrm{g}
$$

and

$$
T_{m}^{0}=\text { const }=580 \mathrm{~K}
$$

The results for the calculation of $g_{0}+2 g_{2} w$ from eq. (8), using $T_{m}^{0}$ and $\Delta h_{g}^{0}$ as given here, are presented in Figure 3.

Consistent sets of $g_{0}$ and $g_{2}$ values, which were calculated from Figure 3 , are given in Table II. As can now be seen from Figure 3 and from Table II there is an increasing concentration dependence of $g$ for the solvent series studied. Again one should realize that only for toluene as a solvent have we shown ${ }^{1}$ that $g_{1} / T$ is zero. For other solvents, $g_{2}$ as given in Table I may well contain enthalpic contributions, originating from the term $g_{1} / T$ (or even from higher contributions ${ }^{11}$ such as $g_{4} \ln T$ ).

The concentration dependence of $g$ values for solutions of PPO in toluene as well as in the other solvents can be explained by equation-of-state theories, as developed by Prigogine ${ }^{9}$ and Flory. ${ }^{10}$ Table III shows the results for a calculation of $g_{v}$, the concentration-dependent part of the interaction parameter $g$, from an easy to handle modification by Dayantis ${ }^{15}$ of Flory's expression ${ }^{10}$ for the residual entropy:

$$
\Delta S^{R}=-R\left[w_{0} \ln \left(\frac{\tilde{v}_{0}^{1 / 3}-1}{\tilde{v}^{1 / 3}-1}\right)+w c_{1} \ln \left(\frac{\tilde{v}_{1}^{1 / 3-1}}{\tilde{v}}\right)\right]
$$




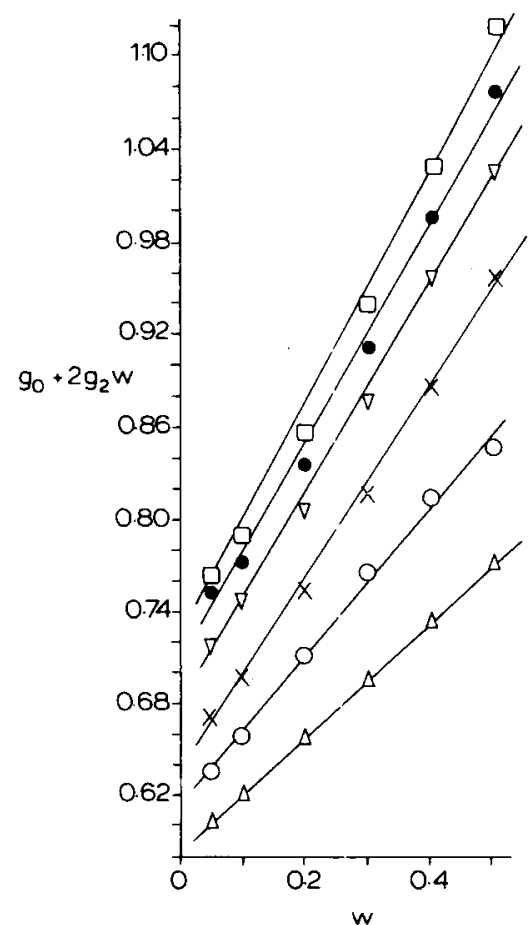

Fig. 3. Calculated curves for $\left(g_{0}+2 g_{2} w\right)$ with the assumption of co-crystallization by the solvents: points as in Figure 1.

where $\tilde{v}_{0}$ and $\tilde{v}_{1}$ are the so-called reduced specific volumes of solvent and polymer, respectively, $c_{1}$ represents the number of degrees of freedom of the polymer segment compared to the solvent, and $\tilde{v}$ is the reduced specific volume of the solution. Now the concentration-dependent part of the $g$ parameter is calculated from ${ }^{10,15}$

$$
g_{v}=-\Delta S^{R} / R w_{0} w
$$

For details of the calculation see the Appendix.

When the $g_{v}$ of Table III are plotted against $w$, a straight line is obtained. Its slope is $g_{2}$; for toluene it was calculated to be 0.16 . In the same way, $g_{2}$ values for the other solvent-PPO systems have been calculated. The intercepts of the $g_{v}$ plots are not equal to $g_{0}$; an enthalpic, supposedly concentration-independent, contribution $g_{h}$ has to be added: $g_{0}=\left(g_{v}\right)_{0}+g_{h}$. (The

TABLE II

Calculated $g_{0}$ and $g_{2}$ Values with the Assumption of Cocrystallization from the Solvent

\begin{tabular}{lccc} 
& $g_{0}$ & $g_{2}$ & $\begin{array}{c}\text { Correlation coefficient of } \\
\text { curve }\end{array}$ \\
\hline Ethylbenzene & 0.61 & 0.25 & 0.997 \\
Propylbenzene & 0.63 & 0.32 & 0.998 \\
Butylbenzene & 0.67 & 0.35 & 0.998 \\
Pentylbenzene & 0.70 & 0.38 & 0.998 \\
Hexylbenzene & 0.71 & 0.41 & 0.997 \\
\hline
\end{tabular}


TABLE III

Calculated Values of the Concentration-Dependent Part $g_{v}$ from Simplified Equation-ofState Theory, for the Toluene-PPO System ${ }^{\mathrm{a}}$

\begin{tabular}{cc}
\hline$w$ & $g_{v}$ \\
\hline 0.05 & 0.428 \\
0.1 & 0.435 \\
0.2 & 0.450 \\
0.3 & 0.466 \\
0.4 & 0.483 \\
0.5 & 0.502 \\
\hline
\end{tabular}

a Assumptions: $\tilde{v}=1.3947, \tilde{v}_{1}=1.1653, c_{1}=0.125$ (see Appendix for details).

assumption that this enthalpic contribution does not depend on the concentration would imply that only dispersion forces are present. ${ }^{16}$ The polar forces could then be accomodated independently in the concentration dependent term.)

We have calculated the $g_{h}$ from solubility parameter theory ${ }^{16}$ :

$$
g_{h}=\left(V_{0} / R T\right)\left(\delta_{d}^{0}-\delta_{d}^{\prime}\right)^{2}
$$

using the $\delta_{d}^{\prime}$ value for PPO from Koenhen and Smolders ${ }^{16}$ and taking $\delta_{d}^{0}$ values from van Krevelen. ${ }^{8}$ The results, combined with the results for $g_{2}$ and $g_{0}$, are presented in Table IV.

\section{DISCUSSION}

\section{Interaction Parameters in Solution}

The results for $g_{0}+2 g_{2} w$ vs. $w$ calculated with eq. 8 and $\Delta h_{g}^{0}=11.84$ $\mathrm{cal} / \mathrm{g}$ give values for the series of solvents (Fig. 2) which are contrary to expectations, and therefore we believe that these values are not correct.

When the assumption was made that the solvent forms part of the crystals and the simple dependence of $\Delta h_{u}^{0}$ on $M_{u} / M_{0}$ was introduced, straight lines were obtained for $g_{0}+2 g_{2} w$ vs. $w$, which permitted calculation of consistent $g_{0}, g_{2}$ pairs for each solvent. It must be stressed, however, that it is in no way proved that the dependence we used has to be the correct one, nor is the constancy of $T_{m}^{0}$ experimentally proved.

The linear dependence of $g$ on $w$ (for $0 \leq w \leq 0.5$ ) is in accordance, however, with equation-of-state theory, as is shown in Table III. The substantial dependence of $g_{2}$ on solvent molecular weight (Table II) is not predicted by applying this theory in the way we did. On the other hand, from the solubility parameter theory in its simplest form [eq. (14)] using only contributions from dispersion forces and thereby ignoring concentration dependence (and thus a possible contribution to $g_{2}$ ), it is shown that the enthalpic contribution to the interaction parameter $g$ increases with increasing molecular weight of the solvent (Table IV). Thus, equation-ofstate theory and (simple) solubility parameter theory combined correctly predict the observed increase in $g_{0}$ from toluene to $n$-hexylbenzene.

We also see from Table IV, that $g_{h}$ for toluene solutions is 0.06 . As $g_{h} \equiv$ 
TABLE IV

Calculated Values for the Enthalpic Interaction Parameter $g_{h}$; Calculated and Experimental ${ }^{a}$ Values for $g_{0}$ and $g_{2}$

\begin{tabular}{|c|c|c|c|c|c|c|}
\hline & \multirow[b]{2}{*}{$\delta_{d}^{0}$} & \multirow[b]{2}{*}{$g_{h}$} & \multicolumn{2}{|c|}{$\boldsymbol{g}_{0}$} & \multicolumn{2}{|c|}{$g_{2}$} \\
\hline & & & Calculated & Experimental & Calculated & Experimental \\
\hline Toluene & 8.84 & 0.058 & 0.48 & 0.58 & 0.16 & 0.19 \\
\hline Ethylbenzene & 8.76 & 0.087 & 0.50 & 0.61 & 0.15 & 0.25 \\
\hline$n$-Propylbenzene & 8.73 & 0.108 & 0.52 & 0.63 & 0.14 & 0.32 \\
\hline$n$-Butylbenzene & 8.69 & 0.136 & 0.54 & 0.67 & 0.14 & 0.35 \\
\hline$n$-Pentylbenzene & 8.66 & 0.163 & 0.56 & 0.70 & 0.13 & 0.38 \\
\hline$n$-Hexylbenzene & 8.63 & 0.19 & 0.59 & 0.71 & 0.13 & 0.41 \\
\hline
\end{tabular}

a Experimental values from Table II.

$-T(\partial g / \partial T)=g_{1} / T$, this means that $g_{1} / T$ is calculated to be about 0.06 . In the same way, we obtain a value of about 0.20 for $g_{1} / T$ for $n$-hexylbenzene.

This casts doubt upon our assumption that $g_{1} / T$ vanishes for the whole series of solvents, and for the whole range of temperatures. We did not pursue this matter further, however, because simple solubility parameter theory predicts $g_{h}$ to be independent of temperature, whereas $g_{h}$ is known to depend strongly on temperature. ${ }^{19}$ It can even become negative at high temperatures, ${ }^{19}$ so $g_{1} / T$ is certainly not the only term that accounts for the dependence of $g$ on $T$. Second, $g_{h}$ should not only be calculated, but measured as well (calorimetrically).

Thus, as has been anticipated above, our assumption that $g_{1} / T=0$ for all solvents at all temperatures implies a systematic error in $g_{2}$. More involved calculations, ${ }^{19}$ and calorimetric measurements, are needed to account quantitatively for this error.

\section{Enthalpy and Temperature of Fusion; Solvent Cocrystallization}

From the melting-point depression data of the system PPO-toluene we obtained, through the application of eq. (8), optimized $\Delta h_{g}^{0}$ and $T_{m}^{0}$ values of $11.84 \mathrm{cal} / \mathrm{g}$ and $580 \mathrm{~K}$, respectively, which differ from those reported previously by ourselves ${ }^{1}$ and by other workers. ${ }^{20-22}$ This difference mainly stems from the optimization procedure: the present melting-point depression data for the system PPO-toluene are, in fact, in excellent agreement with data published previously (see also Fig. 6 of ref. 1, where our previous data are compared to those obtained by Shultz and $\mathrm{McCullough}^{20}$ ).

The main point of difference is that we optimized $\Delta h_{g}^{0}$ and $T_{m}^{0}$ using experimental data for the $g$ parameters $\left(g_{0}, g_{1} / T=0\right.$, and $\left.g_{2}\right)$. In our opinion it is not correct to optimize $\Delta h_{g}^{0}$ and the $g$ parameters using an experimental value for $T_{m}^{0}$ as we did previously ${ }^{1}$ and the other workers ${ }^{20-22}$ did as well (with $T_{m}^{0}$ values in the range of $510-545 \mathrm{~K}$ ). It is implicit in the thermodynamic derivation [eqs. (2)-(8)] that $\Delta h_{g}^{0}$ and $T_{m}^{0}$ refer to hypothetical values for fully crystallized polymeric units. This being so, no experimental values for $T_{m}^{0}$ should be inserted. It is encouraging to find that our optimized (hypothetical) $T_{m}^{0}$ of $580 \mathrm{~K}$ is in the range of $T_{m}^{0}$ values determined experimentally for well-developed spherulitic PPO crystals $^{23}$ : 560-585 K.

Unfortunately, for lack of independently determined $g$ values for the 
systems in question, we cannot apply our procedure to other melting-point depression data from the literature, e.g., to those for the system PPOmethylene chloride. ${ }^{21}$ The value of $\Delta h_{g}^{0}=11.7 \mathrm{cal} / \mathrm{g}$ obtained by Shultz and $\mathrm{McCullough}^{21}$ for this system appears to compare favorably with our value of $11.84 \mathrm{cal} / \mathrm{g}$ obtained for the PPO-toluene system, but this may be fortuitous. Not only did they follow a different optimization procedure, but they also started from the simple Flory equation [eq. (7) above, with the right-hand side multiplied by $M_{0} / M_{u}$ ].

Janeczek et al. ${ }^{22}$ followed a procedure close to our present one, except for the insertion of a $T_{m}^{0}(510 \mathrm{~K})$. When we insert our $T_{m}^{0}(580 \mathrm{~K})$ into their equation, and correct the resulting $\Delta h_{g}^{0}$ by multiplying it by $M_{u} / M_{0}$, we calculate from their data for the PPO-decalin system $\Delta h_{g}^{0}=12.37 \mathrm{cal} / \mathrm{g}$ (whereas their $\Delta h_{g}^{0}$, corrected for $M_{u} / M_{0}$, would read $10.7 \times 120 / 138=$ 9.30). Thus inserting a higher $T_{m}^{0}$ into the Flory-Hoffman equation (applied by Janeczek et al. ${ }^{22}$ ) leads to a higher $\Delta h_{g}^{0}$, whereas insęrting a higher $T_{m}^{0}$ into the simple Flory equation leads to a lower $\Delta h_{g}^{0}$, as Shultz and McCullough showed. ${ }^{21}$

In summary: values for $\Delta h_{g}^{0}$ extracted from melting-point depression data depend strongly on the type of theoretical expression describing the data, as well as on the optimization procedure: either one could optimize $T_{m}^{0}$ and $\Delta h_{g}^{0}$ using experimental $g$ parameters, or one could optimize $g$ parameters and $\Delta h_{g}^{0}$ using an experimental $T_{m}^{0}$. We applied the latter procedure "afterward," to the solutions of PPO in ethylbenzene to $n$-hexylbenzene, with $\Delta h_{g}^{0}$ and $T_{m}^{0}$ optimized from toluene data first. Also, we prefer the Flory-Hoffman equation [eq. (8) above] as the theoretical expression.

Our crude model for solvent incorporation, eq. (9) or (10), is a first approximation only, based on the assumption that solvent molecules of the homologous series toluene to $n$-hexylbenzene do incorporate into the crystal lattice. We justified this assumption by referring to literature data concerning incorporation of other solvents, ${ }^{5,22}$ and did not make independent attempts to determine $T_{m}^{0}$ of pure PPO or PPO/solvent crystals, nor to determine the amount of solvent (if any), resent in the solid PPO masses obtained during our melting-point depression measurements. Nevertheless, eq. (9) works quite well as a means of obtaining reasonable $g$ parameters from melting-point depression data, as has been shown in the previous section.

\section{APPENDIX}

For Table III, we calculated $\Delta S^{R}$ [eqs. (10) and (11)] assuming for $c_{1}$ Dayantis's ${ }^{15}$ value of 0.125 , and for $\tilde{v}$ simple linear additivity ${ }^{15}: \tilde{v}=w_{0} \tilde{v}_{0}+w \tilde{v}_{1}$. The $\tilde{v}_{0}$ and $\tilde{v}_{1}$ have been calculated from solvent and polymer specific volumes $\left(v_{0}=1.155\right.$ and $v_{1}=0.877 \mathrm{~cm}^{3} / \mathrm{g}$, respectively) and from solvent and polymer core volumes $v^{*}\left(\tilde{v}_{0}=v_{0} / v_{0}^{*}\right.$ and $\left.\tilde{v}_{1}=v_{1} / v_{1}^{*}\right)$. The $v^{*}$ have been calculated from a group contribution theory ${ }^{17}$ using the relation $v^{*}=15.17 b r^{\prime}$, with $b=1.28$ and $r^{\prime}$ the (molecular) volume parameter calculated from group contributions as indicated for instance by Oishi and Prausnitz. ${ }^{17}$ The group parameters have been derived from a table given by Gmehling et al.18; for the unknown aromatic ether-oxygen in PPO we had to resort to the aliphatic one in diisopropylether (group no. 27, Table IV of ref. 18). Thus, for Table II the parameters were $c_{1}=0.125, \tilde{v}_{0}=1.3947$, and $\tilde{v}_{1}=1.1653$. 


\section{References}

1. D. M. Koenhen and C. A. Smolders, J. Polym. Sci. A-2, 15, 155 (1977).

2. D. M. Koenhen and C. A. Smolders, J. Polym. Sci. A.2, 15, 167 (1977).

3. D. M. Koenhen, C. A. Smolders, and M. Gordon, J. Poly. Sci. C, 61, 93 (1978).

4. J. Brandrup and E. H. Immergut, Eds. Polymer Handbook, 2nd ed., Interscience, New York, 1975.

5. W. Wenig, R. Hammel, W. J. MacKnight, and F. E. Karasz, Macromolecules, 9, 253 (1976).

6. P. J. Flory, Principles of Polymer Chemistry, Cornell University, Ithaca, NY, 1953.

7. Th. G. Scholte, J. Polym. Sci. A-2, 8, 841 (1970).

8. D. W. van Krevelen, Properties of Polymers, Elsevier, Amsterdam, 1972.

9. I. Prigogine, The Molecular Theory of Solutions, Interscience, New York, 1957.

10. P. J. Flory, Discuss. Faraday Soc., 49, 7 (1970).

11. R. Koningsveld and A. J. Staverman, J. Polym. Sci. A-2, 6, 325 (1968).

12. L. Mandelkern, Crystallization of Polymers, McGraw-Hill, New York, 1964.

13. J. D. Hoffman, J. Chem. Phys., 28, 1192 (1958).

14. J. B. Helms, Dissertation, Groningen, 1970.

15. J. Dayantis, J. Polym. Sci. C, 39, 35 (1972).

16. D. M. Koenhen and C. A. Smolders, J. Appl. Polym. Sci., 19, 1163 (1975).

17. T. Oishi and J. M. Prausnitz, Ind. Eng. Chem. Proc. Res. Dev., 17, 333 (1978).

18. J. Gmehling, P. Rasmussen, and A. Fredenslund, Ind. Eng. Chem. Proc. Res. Dev., 21, 118 (1982).

19. I. C. Sanchez and R. H. Lacombe, Macromolecules, 11, 1145 (1978).

20. A. R. Shultz and C. R. McCullough, J. Polym. Sci. A-2, 10, 307 (1972).

21. A. R. Schultz and C. R. McCullough, J. Polym. Sci. A-2, 7, 1577 (1969).

22. H. Janeczek, E. Turska, T. Szekely, M. Lengyel, and F. Till, Polymer, 19, 85 (1978).

23. A Packter and K. A. Sharif, J. Polym. Sci. B, 9, 435 (1971).

Received December 13, 1983

Accepted June 4, 1984 